\section{T cells go universal}

\section{By Tracey Baas, Senior Editor}

National University of Singapore and St. Jude Children's Research Hospital team has engineered a $\mathrm{T}$ cell therapeutic that can engage in antibody-dependent cell cytotoxicity and enhance the effects of therapeutic antibodies regardless of the target tumor antigen. ${ }^{1}$ The researchers showed proof of concept for boosting the efficacy of multiple marketed cancer antibodies.

They now plan to test $\mathrm{T}$ cell and antibody combinations in clinical trials in which antibody monotherapy did not generate remissions.

To create the $\mathrm{T}$ cell therapy, the team first designed a chimeric receptor that binds to the Fc portion of human antibodies and also delivers $\mathrm{T}$ cell activation signals.

The concept is similar to a chimeric antigen receptor (CAR) T cell, but rather than targeting a cancer-associated antigen, the NUS-St. Jude product targets the Fc portion of a human antibody that binds to a cancer-associated antigen.

Thus, with CAR-based T cells, the variability is in the $\mathrm{T}$ cell therapeutic. In contrast, the variability for the new chimeric receptor T cells is in the antibody therapeutic. As a result, only one type of engineered $\mathrm{T}$ cell is needed to treat multiple types of cancer, which simplifies manufacturing logistics.

The binding portion of the chimeric receptor was modeled after Fc $\gamma$-receptor III (CD16; FCGR3), which is found on NK cells and induces antibody-dependent cellular cytotoxicity (ADCC) when it engages with antibodies. The team reasoned that $\mathrm{T}$ cells expressing CD16 would engage in ADCC toward the cancer cells and selected a variant of CD16 with high affinity for antibodies.

Indeed, previous findings by a team from France showed that T cells engineered to express a CD16 and FCER1G (Fc fragment of IgE high affinity I receptor for $\gamma$-polypeptide) fusion protein induced ADCC against a cultured human B lymphoblast cell line in the presence of an anti-CD20 mAb. ${ }^{2}$

The signaling portion of the NUS-St. Jude chimeric receptor was made up of $\mathrm{CD} 3 \zeta$ and tumor necrosis factor receptor superfamily member 9 (TNFRSF9; 4-1BB; CD137), which induce T cell activation, proliferation and antitumor activity in T cells expressing antigen-specific CARs. ${ }^{3}$

The resulting product was $\mathrm{T}$ cells that express a universal chimeric receptor that targets the constant region of the Fc portion of antibodies.
The team first used a retroviral vector to express the CD16-BB- $\zeta$ construct-shorthand for the part CD16, part 4-1BB, part CD3 $\zeta$ construct-in human peripheral blood $\mathrm{T}$ lymphocytes and tested the cells' ability to enhance the efficacy of Rituxan rituximab against B cell malignancies.

Rituxan/MabThera, an antibody against CD20 from Biogen Idec Inc. and the Genentech Inc. unit of Roche, is marketed for a variety of autoimmune diseases and hematological cancers.

In a cultured human B lymphoblast cell line, Rituxan plus CD16-BB- $\zeta$ expressing $\mathrm{T}$ cells led to $\mathrm{T}$ cell activation, exocytosis of lytic granules and $\mathrm{T}$ cell proliferation, and the combination killed more than $50 \%$ of the target cells within 4 hours.

Importantly, the engineered $\mathrm{T}$ cells did not do anything in the absence of either the antibody or the antigen.

The next step was showing that the CD16BB- $\zeta$-expressing $\mathrm{T}$ cells could target more than one tumor antigen. Thus, the team tested the $\mathrm{T}$ cell and antibody combination in human breast and cancer cell lines expressing HER2 (EGFR2; ErbB2; neu) or human neuroblastoma and osteosarcoma cell lines expressing GD2, which is a target expressed on tumors of neuroectodermal origin.

Indeed, CD16-BB- $\zeta$-expressing $\mathrm{T}$ cells plus Herceptin trastuzumab, which targets HER2, led to ADCC and cancer cell death. Similarly, CD16-BB- $\zeta$-expressing $\mathrm{T}$ cells plus hu14.18K322A, an antibody from St. Jude that targets GD2, led to ADCC and cancer cell death. Genentech markets Herceptin to treat breast and gastric cancers. St. Jude's antibody is in Phase I testing to treat neuroblastoma, melanoma and osteosarcoma.

T cells expressing CD16-BB- $\zeta$ induced significantly higher levels of ADCC than T cells expressing CD16 alone or those expressing the CD16-FCER1G fusion protein $(p<0.0001)$, as well as much more vigorous $\mathrm{T}$ cell expansion.

The strong in vitro results held up in mouse studies. In immunodeficient animals with human B lymphoblast cells, Rituxan followed by the engineered $\mathrm{T}$ cells resulted in remission in all 5 treated mice, whereas none of the 12 mice receiving engineered $\mathrm{T}$ cells or Rituxan alone survived.

Similar results were seen in immunodeficient mice with human neuroblastoma cells that received hu14.18K322A followed by the engineered T cells.

Because dramatic clinical results have been achieved with CARbased cell therapies, the team set out to compare the cancer cell-killing potential of their universal CD16-BB- $\zeta$ T cells with that of CAR T cells that target CD19 and contain the same $\mathrm{T}$ cell signaling molecules.

Again, they tested the strategies in the cultured human B lymphoblast cell line, which expresses high levels of CD19 in addition to CD20. 
In the cultured human B lymphoblast cell line, Rituxan plus CD16BB- $\zeta$-expressing $\mathrm{T}$ cells showed significantly greater cytotoxicity than Rituxan plus CD19-BB- $\zeta$-expressing T cells $(p<0.001)$.

Results were published in Cancer Research.

\section{Moving to man}

The team plans to test its approach in patients with B cell malignancies who previously received antibody therapy but are not in remission.

Instead of virus vector-mediated expression of the construct, the team plans to use electroporation to express its $\mathrm{CD} 16-\mathrm{BB}-\zeta$ construct in the initial trials. "Electroporation adds an extra layer of safety to our system because the T cells will transiently express the construct," said team leader Dario Campana, a professor of pediatrics at NUS. "As a second layer of safety, if any complications are seen, we can stop delivery of the immunotherapeutic antibody and ADCC will be abolished."

The paper already showed that using electroporation is possible.

But other scientists suggested that the main safety concerns are on- or off-target effects of the antibodies that are selected for use with the engineered T cells.

"The new strategy's specificity is only as good as the selectivity of the antibodies used. If these antibodies bind to antigens on nontumor tissues, it raises concerns about toxicity," said Charles Sentman, a professor of microbiology and immunology at the Geisel School of Medicine at Dartmouth

"The results indicate that universal Fc-CAR engineered T cells can significantly enhance ADCC potency and antitumor efficacy of antibody therapies. Potential safety concerns associated with such enhanced potency leading to increases in 'on-target off-tumor' toxicity directed toward normal tissue needs to be further evaluated," added Madhusudan Peshwa, EVP of cellular therapies at MaxCyte Inc.

Carl June thinks that the biggest safety issue is whether the CD16-BB- $\zeta$ construct expressed on the T cells will also be triggered by circulating IgG that is not antigen bound in vivo.

IgG is the main type of antibody found in blood and extracellular fluid and can be induced by infections or cancer.

"The experiments were conducted in immunodeficient mice that had no circulating antibodies, so there is not yet sufficient preclinical toxicology studies available to know if this strategy will be able to move forward to human studies," said June.

June is a professor in the Department of Pathology and Laboratory Medicine at the Perelman School of Medicine at the University of Pennsylvania and director of the translational research program at the Abramson Family Cancer Research Institute at the University of Pennsylvania.

"Binding of patient immune complexes to engineered CD16-BB- $\zeta$ expressing T cells could potentially activate the T cells, resulting in killing of nearby normal cells and induction of inflammation which will not be directed against the targeted tumor," added Sentman. "How significant this would be is unclear, but it is a potential risk that would need to be assessed."
The problem, said Sentman, is that it is not obvious how to go about assessing that risk. "Human Fc receptors do not bind as well to many mouse IgGs, so testing in a mouse model may or may not be that informative," he said.

One approach, said Peshwa, would be to use immunodeficient mice co-infused with human IgGs and then test the antibody and T cell combination.

"T cells being triggered by circulating IgG is very unlikely," said Campana. "Unbound antibody, such as circulating IgG, does not cause receptor cross-linking and hence should not trigger anything."

Safety issues aside, Michael Gladstone, an associate in the life sciences group at Atlas Venture, said that the strategy "allows the unique opportunity to titrate the antibody dosing in a way that is virtually impossible to do with conventional CAR T cells that have a tumor-targeting protein locked in."

The precision comes from the ability to dose specific amounts of antibodies. In contrast, it is difficult to predict how much expansion CAR T cells will undergo.

Gladstone said that the NUS-St. Jude technology is not entirely dissimilar from another type of immunotherapyantibody-drug conjugates. Although virtually all antibody-drug conjugates employ some toxic payload, the academics essentially are using $\mathrm{T}$ cells as the payload, albeit without an actual chemical linker to the antibody.

"When you look at how many parties are trying to devise modular antibody-drug conjugate approaches to increase antibody effectiveness, it's quickly clear how much of a value proposition this type of adaptable approach is," noted Gladstone. "This approach leverages the ultimate effector mechanism: cytotoxic T cells.”

NUS and St. Jude have jointly filed for patents covering the technology, and the IP is available for licensing.

Baas, T. SciBX 6(47); doi:10.1038/scibx.2013.1337

Published online Dec. 12, 2013

\section{REFERENCES}

1. Kudo, K. et al. Cancer Res.; published online Nov. 6, 2013 ; doi:10.1158/0008-5472.CAN-13-1365

Contact: Dario Campana, National University of Singapore, Singapore

e-mail: paedc@nus.edu.sg

2. Clémenceau, B. et al. Blood 107, 4669-4677 (2006)

3. Imai, C. et al. Leukemia 18, 676-684 (2004)

COMPANIES AND INSTITUTIONS MENTIONED

Abramson Family Cancer Research Institute at the University of Pennsylvania, Philadelphia, $\mathrm{Pa}$

Atlas Venture, Cambridge, Mass.

Biogen Idec Inc. (NASDAQ:BIIB), Weston, Mass.

Geisel School of Medicine at Dartmouth, Hanover, N.H.

Genentech Inc., South San Francisco, Calif.

MaxCyte Inc., Gaithersburg, Md.

National University of Singapore, Singapore

Perelman School of Medicine at the University of Pennsylvania, Philadelphia, $\mathrm{Pa}$.

Roche (SIX:ROG; OTCQX:RHHBY), Basel, Switzerland

St. Jude Children's Research Hospital, Memphis, Tenn. 